Gdańsk 2019, Nr. 40

https://doi.org/10.26881/sgg.2019.40.07

Jan Habermehl

Goethe-Universität Frankfurt am Main / Uniwersytet we Frankfurcie nad Menem

\title{
Prinzessin Gräcula. Zum Affen als Figur pädagogischer Reflexion bei Friederike Helene Unger
}

Der Beitrag widmet sich Friederike Helene Ungers kaum bekanntem Feenmärchen Prinzessin Gräcula, das vom gleichnamigen Protagonisten ihres 1804 erschienenen Romans Albert und Albertine erzählt wird. Anhand einer mehrfach verwandelten Affenfigur werden dort zeitgenössische Diskurse angemessener Mädchenerziehung reflektiert. Von der germanistischen Forschung wurde der durchaus produktiven Rezeption der aus der französischen Erzähltradition des 17. Jahrhunderts stammenden weiblichen ,Märchenaffen' in der deutschsprachigen Literatur um 1800 bislang nur wenig Aufmerksamkeit geschenkt.

Schlüsselwörter: Unger, Friederike Helene, Prinzessin Gräcula, Feenmärchen, Mädchenerziehung, Affen als Reflexionsfiguren

Prinzessin Gräcula. On the Monkey as Figure of Pedagogic Reflection in Friederike Helene Unger. This article discusses the little-known fairy-tale Prinzessin Gräcula by Friederike Helene Unger, which is narrated by the eponymous protagonist of her 1804 novel Albert und Albertine. The tale reflects contemporary discourses on appropriate female education through a transformed and retransformed she-monkey character. Up to now, German Studies have paid little attention to the French $17^{\text {th }}$-century narrative tradition of the female 'fairy-tale monkeys' and its reception in the German literature around 1800.

Keywords: Unger, Friederike Helene, Prinzessin Gräcula, German fairy-tales, Girls‘ education, apes and monkeys as reflexive figures

1.

Seit der Antike wird in kulturellen Praktiken die Grenze zwischen Mensch und Tier, Natur und Kultur, Zivilisation und Wildnis und damit jene für die Erfüllung des menschlichen Bedürfnisses nach einer stabilen Identität unverzichtbare Trennung zwischen dem Eigenen und dem Anderen bevorzugt unter Bezug auf die Figur des Affen als menschenähnlichstem Wesen im Raum des Nicht-Menschlichen gesetzt und immer wieder neu justiert. Entsprechend ausführlich wurde das Potential des Affen als Reflexionsfigur anthropologischer und ästhetischer Diskurse bereits aus textwissenschaftlicher und kulturgeschichtlicher Perspektive untersucht, wobei das Hauptaugenmerk auf Affenbilder der Vormoderne oder des 19. Jahrhunderts gelegt wurde (vgl. Janson 1952; Borgards 2009 sowie Griem 2010, weitere Literatur ebd.: 397-427). Das 18. Jahrhundert, in dem Primaten aus Afrika und Asien in großer 
Zahl nach Europa kommen, rückte jedoch bislang nur selten ins Blickfeld. Vor allem dem Nebeneinander zahlreicher populärwissenschaftlicher, zoologischer, anthropologischer, philosophischer, pädagogischer und im engeren Sinne literarischer ,Affentexte', die das recht ungewisse Verhältnis dieser Geschöpfe zum Menschen zu ergründen versuchen, wurde, sieht man von der Vielzahl der Arbeiten zu Rousseaus einschlägigem Discours sur l'inégalité einmal $a b$, von der Forschung bisher nur wenig Aufmerksamkeit gewidmet.

Die folgenden Ausführungen werden dementsprechend nicht zum wiederholten Male die enge Verbindung diverser zeitgenössischer Erziehungslehren mit anthropologischen Konzepten rekapitulieren, sondern sich auf die literaturwissenschaftliche Analyse eines deutschsprachigen Feenmärchens beschränken, in dem der Erziehungs- und Initiationsprozess der Protagonistin anhand einer mehrfach verwandelten Affenfigur erzählt wird. Gezeigt werden soll einerseits, wie sich literarische Texte zur Illustration solcher Prozesse an Affenvorstellungen der Zeit bedienen und diese gleichzeitig aus- und mitgestalten, während andererseits die literarhistorische Darstellung der Rezeption der französischen Märchentradition um 1700 in der deutschen Literatur um 1800 um ein bisher unbeachtet gebliebenes Rezeptionszeugnis ergänzt werden soll.

Vorgestellt wird das Märchen Prinzessin Gräcula von Friederike Helene Unger, das vom gleichnamigen Protagonisten ihres Romans Albert und Albertine im Rahmen eines dilettierenden ,Literaturclubs' an drei Abenden erzählt wird (Unger 1804: 63-124). Auf eine erste Kontextualisierung des Textes und seiner Verfasserin in der deutschsprachigen Literatur des frühen 19. Jahrhunderts und eine kurze Zusammenfassung der bisherigen Forschungsperspektiven folgen eine Skizzierung des Märchenplots sowie die Analyse der titelgebenden Äffin Gräcula, insbesondere im Hinblick auf ihre französische Vorgängerin, die Äffin Babiole aus Madame d'Aulnoys gleichnamigem Feenmärchen aus dem Jahr 1698.

2.

Zunächst soll allerdings eine knappe Rekapitulation der Affenbilder der abendländischen Kulturgeschichte die Konzeption der Affenfigur in Ungers Märchen verdeutlichen. Es lassen sich grosso modo drei Traditionslinien nachzeichnen. Zum einen wirkt die aus dem Physiologus stammende Auffassung des Affen als figura diaboli noch bis ins 19. Jahrhundert nach. Der Teufel gilt als erfolgloser Nachäffer Gottes, und in ein ähnliches Verhältnis, nun eben zum Menschen, wird auch der Affe gerückt, dessen Mimikry nur eine oberflächliche ist. Da den Tieren neben dem Hang zur Völlerei auch eine ausschweifende, monströse, gewissermaßen teuflisch entfesselte Sexualität unterstellt wird, bilden sie die Kontrastfigur zur christlichen Tugendlehre.

Daneben existiert seit der Antike auch noch eine satirische, auf den unterstellten Nachahmungstrieb anspielende Interpretation des Tieres, aus der sich im späten Mittelalter der

1 Greifbar ist der französische Text in einer jüngeren Gesamtausgabe: Madame d'Aulnoy (2004: 507-533). Babiole wurde in jüngerer Zeit ausschließlich im angelsächsischen Raum in den Blick genommen: Hoffman (2005) erläutert den Zusammenhang des Märchens mit realen ,Affenmädchen' des 17. Jahrhunderts und die Bedeutung von ,Wundern' für höfische Reputation und Repräsentation, während Birberick (2011) den Text aus Perspektive der Gender Studies analysiert und Seifert (2011) sich in ähnlicher Herangehensweise auf die Unterschiede zwischen männlicher und weiblicher Mensch-Tier-Hybridität konzentriert. 
Topos des gelehrten Affen herausbildet. Diese Figur karikiert einen ,närrischen' und wenig originellen Intellektuellen, der seinen Lehrern zwar nachzueifern versucht, ohne aber über die bloße Nachahmung hinausgehen zu können. Indem jedoch in der Frühen Neuzeit unter dem Schlagwort ars simia naturae der die Natur, nachäffende Künstler mit dem Tier in Verbindung gebracht wird, wird der Topos des gelehrten, dichtenden Affen in ein positives Licht gerückt. Das Tier wird zur figura poetae schlechthin. Diese Aufwertung führt dazu, dass der schreibende Affe seitdem nicht mehr nur als Figuration akademischer ,Narrheit' auftritt, sondern nun selbst Kritik an gesellschaftlichen Verhältnissen üben kann. ${ }^{2}$

Eine dritte und für Ungers Märchen leitende Figuration des Affen ließe sich als figura educandi bezeichnen, die auf die bereits angesprochene viel diskutierte Frage nach der Möglichkeit einer menschlichen Erziehung von Affen Bezug nimmt. Exemplarisch dienen Jean-Jacques Rousseau und der prominente Naturkundler Leclerc de Buffon als Vertreter einander entgegengesetzter Positionen, die auf die unterschiedliche taxonomische Verortung jener schwanzlosen Geschöpfe, die heutzutage als Menschenaffen bezeichnet werden, zurückzuführen ist. Einig sind sich beide in der Auffassung, dass Affen mit Schwanz, also Meerkatzen und andere kleinere Primaten, den Tieren zuzurechnen sind, was auf das antike Konzept der scala naturae alludiert. Rousseau und Buffon geht es letztlich um den Orang-Utan, der in der zeitgenössischen Theoriebildung entweder als Tier, als Monster im Sinne einer Chimäre aus Mensch und Tier oder als, unvollkommener' Mensch, als, l'homme sauvage' begriffen werden kann (Borgards 2009: 294-295). Buffon postuliert in seiner Naturgeschichte der vierfüßigen Thiere eine unüberbrückbare Distanz zwischen Affe und Mensch: „Es ist also der Affe [...] in der Wahrheit nur ein bloßes Thier, das äußerlich die Maske der menschlichen Figur an sich trägt, dem aber innerlich der Gedanke fehlt, dem alles fehlt, was den Menschen macht" (Buffon 1791: 168). Erziehung im Sinne einer fortwährenden Vervollkommnung der Sprach- und damit der Vernunftfähigkeit sei beim Affen daher unmöglich, so Buffon.

Rousseau nähert sich in seinem Discours der Beantwortung der Frage nach der Erziehung von Menschenaffen nicht, wie zu erwarten wäre, über ein pädagogisches Experiment, das womöglich die Fähigkeit zur Perfektibilität bei Orang-Utans aufzeigte, sondern über ein zoologisches Kreuzungsexperiment:

Es gäbe jedoch ein Mittel, durch das sich, falls der Orang-Utan oder andere der menschlichen Art zugehörten, die krudesten Beobachter sogar anhand eines augenfälligen Nachweises hierüber Gewissheit verschaffen könnten; aber abgesehen davon, dass eine einzige Generation für dieses Experiment nicht ausreichte, weil das, was nur eine Annahme ist, als wahr nachgewiesen sein müsste, bevor der Versuch, der die Tatsachen bestätigen sollte, frei von Schuld gewagt werden könnte. (Rousseau 1997: 333)

Die Schlussaporie der Undurchführbarkeit des Versuchs, der das Kreuzungs- zu einem reinen Gedankenexperiment macht, öffne, so Roland Borgards, einen fiktionalen Raum (2009: 298). Die Grenze zwischen Tier und Mensch lässt sich nicht eindeutig bestimmen, es bleibt nur der Raum des Experiments, in dem sie potentiell verlaufen könnte. Literarische Affentexte, darunter Ungers Märchen, situieren sich in diesem Raum.

\footnotetext{
2 Erinnert sei beispielsweise an die ,Affenbriefe` von Rétif de la Bretonne, E. T. A. Hoffmann oder Franz Kafka.
} 
3.

Zur Leipziger Ostermesse des Jahres 1804 erscheint beim einflussreichen und von hochrangigen Zeitgenossen geschätzten Berliner Verleger Johann Friedrich Unger der anonyme Roman Albert und Albertine. Der Roman stammt, wie wir heute wissen, aus der Feder von Ungers Ehefrau Friederike Helene (vgl. zu Person und Werk v.a. Lehmstedt 1996 und Giesler 2003). Vermutlich 1741 in Berlin geboren, ist sie ab 1782 als Schriftstellerin und Herausgeberin von Almanachen und literarischen Journalen für ein dezidiert weibliches Lesepublikum sowie als Übersetzerin aus dem Englischen und dem Französischen tätig. Insbesondere als Übersetzerin von Rousseau tritt Unger hervor. Einige ihrer teilweise recht erfolgreichen und zum Teil sehr lobend rezensierten Texte publiziert sie unter eigenem Namen, anderes erscheint anonym. Nach dem Tod ihres Mannes im Jahr 1805 übernimmt sie das zwar prosperierende, aber hoch verschuldete Verlagshaus, kann dessen Niedergang in den Jahren der französischen Besatzung jedoch nicht mehr abwenden. Verarmt und weitestgehend vergessen stirbt sie im folgenden Jahr im Berlin.

Ungers, einsames Ende` steht in starkem Kontrast zu ihrer herausragenden Stellung in der kulturellen Landschaft um 1800. Ob sie, wie es in der noch übersichtlichen Forschung hin und wieder kolportiert wird, als Initiatorin eines literarischen Salons nach französischem Vorbild bezeichnet werden kann, ist der derzeitigen Quellenlage nach nicht mit Gewissheit festzustellen. Zumindest die Autoren des Verlagshauses versammelt Unger um sich, unter denen sich auch - in Person von Ludwig Tieck und den Schlegel-Brüdern - die ,shooting stars' der Frühromantik befinden. Zudem steht sie in regem Briefkontakt mit anderen Verlegern, Gelehrten, Künstlern und Schauspielern und ist mit den zeitgenössischen ästhetischen Diskursen sowie aktuellen philosophischen und pädagogischen Diskussionen gut vertraut. In geistesgeschichtlicher Hinsicht befindet sich Friederike Helene Unger gewissermaßen ,auf der Höhe der Zeit'. Dennoch ist sie keine Avantgardistin. Ihr eigenes poetologisches Programm verbindet rationalistisch-didaktische, klassizistisch-formstrenge und sozialkritisch-satirische Momente und rückt sie so in eine Position zwischen Aufklärung, Weimarer Klassik und Frühromantik, wobei sie die ästhetische Radikalität eines Friedrich Schlegel etwa strikt ablehnt. Als Beispiel des für Unger charakteristischen ,Dazwischen ließe sich der Roman Albert und Albertine anführen, den die Herausgeberin des Nachdrucks Susanne Zantop als „literarisches Zwitterwesen - halb Liebesroman, halb Literatursatire“ (Zantop 1996: 323) bezeichnet. Satirisch ist der Text insbesondere in Hinblick auf die Frühromantiker, wird doch hier eine literarische Gesellschaft inszeniert, deren dilettierende Mitglieder und ihre schwärmerisch-sentimentale Kunstreligion vom Erzähler verspottet werden.

Zwar ist die Verfasserin über ihren Tod hinaus in bio-bibliographischen Verzeichnissen des 19. Jahrhunderts präsent, in die im engeren Sinne literaturwissenschaftliche Diskussion wurde sie allerdings erst am Beginn des 20. Jahrhunderts von Christine Touaillon eingebracht:

Heute besitzen ihre Romane nur mehr literarhistorischen und kulturgeschichtlichen Wert; letzteren namentlich durch die lebhaften und gegenständlichen Schilderungen der Frauenerziehung im 18. Jahrhundert. Zu ihrer Zeit galt sie für eine wichtige Erscheinung [...]. (Touaillon 1919: 261) 
Dieses Urteil bleibt zunächst bestehen, und der Name Friederike Helene Unger verschwindet beinahe vollständig aus einschlägigen Lexika. Erst im Zuge der feministischen Germanistik der achtziger Jahre wird sie gewissermaßen wiederentdeckt, einige Nachdrucke und erste überblicksartige Arbeiten erscheinen (vgl. Giesler 2003). Geprägt sind die Forschungsbeiträge von einem Aufwertungsnarrativ, das Touaillons Einschätzung zu relativieren versucht, indem beispielsweise in engmaschigen Analysen der zahlreichen intertextuellen Verknüpfungen einzelner Bestandteile des OEuvres dessen außergewöhnliche Literarizität belegt werden soll. Hinzu kommen an jüngeren Perspektiven der Queer Studies orientierte Lektüren, die Unger eine ungeahnte (Post-)Modernität in Genderfragen zuschreiben und sie zu einer die heteronormativen Geschlechterordnungen der sogenannten Goethezeit kritisch hinterfragenden Feministin avant la lettre stilisieren (Giesler 2009). Unger wird zunehmend zu einem Paradebeispiel ,weiblicher' Kreativität um 1800, wobei sich die Untersuchungen jedoch meist auf einige wenige Texte wie etwa ihren Debütroman Julchen Grünthal. Eine Pensionsgeschichte aus dem Jahr 1784 beschränken.

Prinzessin Gräcula wurde bislang meist recht pauschal als Gegenstück zu Ungers ,Bildungsromansatire' Prinz Bimbam, die die Erziehung einer männlichen Märchenfigur erzählt, charakterisiert (vgl. Giesler 2015). Die Affenfigur selbst stieß bislang noch auf kein Interesse der Forschung. Zunächst zum Plot:

Im „weiten Gebiete der Phantasie“ (Unger 1804: 63) liegt das Königreich des kinderlosen Herrscherpaares Fricando, einem ausschließlich an leiblichen Genüssen interessierten „moralischen Krüppel“ (ebd.), und Sentimentale, vom Erzähler als preziöse Ästhetin beschrieben. Um ihrer Kinderlosigkeit ein Ende zu bereiten, beschließt Sentimentale, den Genius Frivolo aufzusuchen, von dem, wie es heißt, „kein einsames Weib [...] einsam“ (ebd.) zurückgekehrt sei. Auf der Rückreise vom erfolgreichen Aufenthalt - die Vaterschaft ist freilich ungeklärt - stößt das Paar auf eine Waldhütte, in der „die erstgebohrne Tochter der Zeit: die Erfahrung “ (ebd.: 76) lebt, die von Sentimentale direkt in ein Gespräch über ihren etwaigen Erben verwickelt wird, worauf die Erfahrung ihr prophezeit: „Deine Tochter wird wunderschön, wunderklug seyn. Machst du sie aber nicht auch wundergut, so wirst du das Orakel, das zu deinen Wünschen Ja! sprach, in die Tiefen des Orkus wünschen“ (ebd.: 76-77). Auf die verlegene Nachfrage der Königin, wie das zu bewerkstelligen sei, erhält sie die Antwort: „Sei es selbst! Lass sie nicht sich in den Irrgarten der Sophisten verirren! Bewahre ihren Fuß vor den Rosengebüschen der Wollust“ (ebd.: 77). Kurz nach der Rückkehr kursieren Gerüchte im Volk, „das Orakel habe der Königin einen leibhaften Affen zum Thronerben gewährt“" (ebd.: 78). Gräcula, so der Name der Erbin, wird geboren und mit allem beschenkt, „was die Schönheit glänzend machen und den Verstand erweitern kann; doch keine dachte an der Gaben schönste: an ein schönes, weibliches Herz, an ein Gemüth, das die Schönheit einzig schön macht, das den Verstand einzig zur schönen Gabe erhöht“ (ebd.: 81-82). An der Stelle des Herzens befindet sich nur ein kalter Kieselstein. Die Prinzessin wird gewissermaßen à la mode erzogen und hört tagtäglich Oden, Elegien oder tiefsinnige Gespräche über philosophische Spitzfindigkeiten, bis sich im Alter von fünfzehn Jahren die Schwestern Wollust und Üppigkeit als Repräsentantinnen Frivolos bei ihr melden, nachdem sie zuvor eine ihr vorgeschlagene Inobhutnahme durch die Erfahrung strikt abgelehnt hatte. Regelmäßige Treffen mit den Schwestern folgen. Nach übermäßigem Alkoholgenuss sinkt Gräcula eines Tages weit entfernt vom elterlichen Schloss in die Arme des Tänzers Salto, mit dem sie kurz darauf entflieht und durch die Lande irrt. Zufällig stoßen beide auf die Waldhütte der Fee Erfahrung, die die Türe öffnet und sie verwandelt: „So werde und sei denn, was du durch dich selbst werden konntest: ein thörichter Affe“ (ebd.: 99-101). Gräcula und Salto werden daraufhin von einer fliegenden Holzkiste auf eine Insel gebracht, die von weiteren in Tiere verwandelten Mitgliedern der höfischen Gesellschaft bevölkert wird, die reihum die Geschichte ihrer Metamorphose berichten. Ihrer Funktion als übernatürliche Lenkerfigur entsprechend wacht die Fee Erfahrung weiterhin über 
Gräcula, die im weiteren Verlauf des Märchens mehrere Tugendproben zu bestehen hat, die dazu führen, dass sie mehrfach ihr äußeres Erscheinungsbild wechselt (Meerkatze, Äffin, Marmorstatue) und unter anderem ihr Kieselsteinherz verliert, ehe sie zum Schluss unter dem neuen Namen Sophia ,an der Hand der Erfahrung, der Weisheit und Freundschaft ihre Staaten zu den glücklichsten [macht], welche je die Sonne beschien" (ebd.: 123-124).

Der Handlungsverlauf folgt recht eng dem Muster der preziös-galanten Feenmärchen französischer Manier, die im 18. Jahrhundert auch im deutschsprachigen Raum von einem breiten Publikum rezipiert werden (vgl. einführend Dammann 1981 und Baader 1986 sowie zur Rezeptionsgeschichte Blamires 2006). Im Zentrum der auf den ersten Blick recht homogenen Textgruppe steht vor allem der Erziehungs- und Bildungsprozess der Protagonistinnen und Protagonisten von der Geburt bis zur Übernahme der Herrschaft. Die zahlreichen erzählten Anthropomorphisierungen von Tierfiguren sowie Transformationen von menschlichen Figuren in hybride Tierwesen sind größtenteils von autoritären Instanzen wie Feen und Zauberern arrangierte und gelenkte Initiationen, die als Prozesse einer Identitätsetablierung verstanden werden können, und die ihren Abschluss in der Übereinkunft von Individualstreben und Gesellschaftsstreben finden. Tugendproben unterschiedlicher Art und meist auch das Scheitern einer Liebesbeziehung zu einem äußerst verführerischen, aber freilich nicht gesellschaftsfähigen Partner komplettieren das gängige Motivarsenal.

Ungers hochartifizielles Märchen steckt erwartungsgemäß voller intertextueller Referenzen und Anspielungen auf zeitgenössische Philosopheme, von denen hier einige kurz vorgestellt werden sollen. Der Name der Protagonistin verweist zwar zweifellos auf den antikisierenden Zeitgeist um 1800, was von der Fee Erfahrung im Dialog mit Sentimentale spöttisch kommentiert wird: „Nun, so weihe denn dein Kind schon durch den Namen zur Thorheit des Zeitalters ein; meinetwegen!“ (Unger 1804: 81). Darüber hinaus lässt sich ein Bezug zu Plinius herstellen, der im 21. Buch seiner Naturgeschichte von einer Rose gleichen Namens berichtet, deren Blätter sich erst öffnen, so sie von menschlicher Hand berührt werden:

Es gibt auch eine andere, sogenannte kleine griechische Rose mit zusammengerollten Blätterbüscheln, die nur mit der Hand gezwungen aufbricht, immer gerade erst aufzusprießen scheint und sehr breite Blätter hat [...]. (Plinius 1999: 29)

Man könnte schließen, dass zur, Vervollkommnung' der inneren Anlagen dieser Blume ein äußeres Zutun erforderlich sei; Parallelen zu pädagogischen Strömungen der Zeit scheinen unverkennbar.

Gräculas erste Laute sind Mozart-Kantilenen, die jedoch im Laufe der ,Stillen Post' vom Hof zu den Vorstädten für Arien aus Karl Friedrich Henslers und Ferdinand Kauers Wiener Singspiel Das Donawweibchen gehalten werden, und ihre ersten Worte, die sie schon kurz nach der Geburt von sich gibt, lauten in bester fichteanischer Manier: „Mama - ich - nicht ich“ (Unger 1804: 85). Die Erziehungsbücher, die ihre Mutter konsultiert, stammen von Rousseau und von Campe, und „zum Noth- und Hülfsbüchlein wird mir auch noch die Frau le Prince de Beaumont zur Hand gehen“ (ebd.: 83), so Sentimentales Überlegungen. Auch ,im weiten Gebiete der Phantasie` werden offensichtlich Feenmärchen als pädagogische 
Literatur rezipiert. ${ }^{3}$ Das Schwesternpaar Üppigkeit und Wollust empfiehlt Gräcula hingegen als Adoleszenzlektüre den mehrbändigen ehekritischen Roman Das Paradies der Liebe des Briten James Lawrence, der erst 1801 ausgerechnet im Verlagshaus Unger erschienen war. Belesen und kulturaffin erscheinen auch die Verwandelten der Insel, die etwa Verse Ewald Christian von Kleists singen und von den Neuangekommenen sofort wissen wollen, „ob der neue Musenalmanach schon erschienen wäre? welche Philosophie jetzt die neuste Mode sei? ob der vierte Theil der Donau-Nymphe schon gegeben worden? [...] ob Wallenstein noch nicht travestirt sei?" (ebd.: 108).

Der ausschlaggebende intertextuelle Verweis, der das gesamte Märchen als Rezeptionszeugnis des bereits erwähnten französischen Vorgängers ausweist, liegt in einem der Namen, die Gräcula von der Fee Erfahrung erhält: Babiole. Bevor die literarhistorischen Hintergründe dieser Namensgebung erhellt werden können, muss zunächst Gräculas,(Un)menschlichkeit` präzisiert werden.

Indem der Erzähler Albert den intradiegetischen, größtenteils weiblichen Zuhörern und damit auch dem Leser jegliche Aussagen über das körperliche Erscheinungsbild der Prinzessin ausschließlich in Form von Gerüchten präsentiert, hält er es gezielt offen, ob Sentimentale nun tatsächlich einen wie auch immer gearteten Affen geboren hat. Selbst unter der Voraussetzung, dass Gräcula kein Affe ist, ist ihre Erziehung trotzdem ausschließlich auf,äffisches' Nachahmen ,vernünftigen 'Verhaltens und Räsonierens ihrer männlichen Lehrer konditioniert. In diesem Fall lassen sich die Gerüchte rund um ihre Geburt nachträglich als zukunftsgewisse Prolepsen lesen. Die Unklarheit, ob die Prinzessin ein gelehrter Affe im ,klassischen Sinn' ist, bleibt bestehen. Sicher ist bloß, dass weibliche Erziehung ohne Herausbildung des ,Wunderguten scheitern muss, weswegen es - feenmärchentypisch - eines verwandelten Zustands bedarf, aus dem heraus die Entwicklung Gräculas zur angestrebten Trias aus ,Wunderschönem', ,Wunderklugem' und ,Wundergutem ' angegangen und vollendet werden kann. Ihre Rückverwandlung erfolgt stufenweise. Sie wird von der Fee zur Strafe für ihre Uneinsichtigkeit zunächst in eine Meerkatze verwandelt. Die Erfahrung rät ihr dennoch: „Verzweifle nicht! Siehe! Beobachte! Lerne! Jeder Grad von Veredlung und Selbsterkenntniß wird dir zugerechnet“ (ebd.: 102). Gräcula beschließt, der Stimme zu folgen und ,aus einer scheußlichen Meerkatze wurde sie ein zierliches Äffchen. Die Stimme rief: ,Wohl dir, Babiole! du siehst, ich halte Wort!““(ebd.). Der erste Schritt zur Vollkommenheit ist also Selbsterkenntnis und führt von der Meerkatze der scala naturae entsprechend zunächst zum schwanzlosen Affen.

Bei Madame d'Aulnoy wird, um die intertextuelle ,Reichweite' des Namenswechsels zu betonen, ebenfalls ein meerkatzenähnliches Schwellenwesen namens Babiole geboren, das jedoch über ein menschliches Herz und eine menschliche Vernunft verfügt, vom Hof allerdings dezidiert als Tier, wenn auch als besonders außergewöhnliches, behandelt wird. Das Märchen schildert im Verlauf der Handlung zwei unterschiedliche Prozesse. Auf der einen Seite stehen der Prozess der Zivilisierung eines angeblichen Affen zum gelehrten, vernünftigen Lebewesen, und damit gleichzeitig der Erziehungsprozess eines als Affe erzogenen verwandelten Mädchens. Die Gegenbewegung zu dieser doppelten Entwicklung ist die stufenweise

3 Jeanne-Marie Leprince de Beaumont (1711-1780) ist heutzutage noch am ehesten als Verfasserin einer prominenten Fassung des Märchens Die Schöne und das Biest in Erinnerung geblieben. 
Selbsterkenntnis und Selbsterfahrung Babioles, die zum gesellschaftskonformen Eheleben führen muss und die in ähnlicher Form auch bei Unger begegnet. Gräcula/Babiole gedenkt auf der Insel ihrer Eltern und spürt an Stelle des kalten Kiesels sofort ein lebendiges Herz in ihrer Brust. Auf der Tierinsel entwickelt sie ihre „gesunde Vernunft“ (ebd.: 113), die die ,nachgeklapperten' philosophischen Maximen ablöst. Weder ein gesteigerter Ästhetizismus, dem Gräculas Mutter anhängt, noch eine reine Vernunfttätigkeit im Sinne Kants - als Kind gibt Gräcula etwa den Kategorischen Imperativ „als selbsterfunden“ (ebd.: 91) aus - können die sich rein aus, natürlicher Erfahrung' speisende, gesunde Vernunft' ersetzen, die für die Erziehung hin zur weiblichen Vollkommenheit allein von Bedeutung sei. ${ }^{4}$

Während des weiteren Aufenthalts auf der Insel erläutert die Fee Erfahrung Babiole ihre Absicht, sie bei menschlicher Sprachfähigkeit in eine Marmorstatue zu verwandeln und zum Schweigen zu verdammen, damit sie lerne, zu dulden: „Höre! Dulde! Schweige! Ein einziger Laut verwandelt dich in kalten Marmor, der du zu seyn scheinst" (ebd.: 116). Die Prinzessin verliert ihren Affennamen Babiole und wird vom Erzähler wieder Gräcula genannt. Als Marmorbildnis steht sie nun im elterlichen Palast und muss sich zuerst den Beurteilungen der Kunstkritiker stellen. Nachdem eine Hofbedienstete die verblüffende Ähnlichkeit der Statue mit der vormaligen Prinzessin erkennt, muss Gräcula anschließend auch noch die Schmähungen des Hofes erdulden. Ein Zuschauer fragt, wer denn an all dem Schuld habe und beantwortet sich die Frage selbst: „[...] die gelehrte Närrin, ihre Mutter, die durchaus ein kleines Wunder haben wollte! Zu der Zeit der Geburt raunte man sich seltsame Dinge zu“ (ebd.: 118-119). Die beleidigenden Gespräche rund um ihre Person werden weitergeführt, ein Zuschauer bemerkt allerdings: „[...] die arme Prinzessin wurde zu viel erzogen. Die schwerfälligste Schmeichelei umgab schon ihre Wiege. Sie hat nie durch die Gelehrsamkeit bis zum Menschen durchdringen können" (ebd.: 120). Irgendwann verliert sich das Interesse an der Statue, und die Erfahrung erscheint eines Tages, Gräcula zu erlösen. Sie sagt: „Sophia, deine Klugheit macht dich fortan dieses Namens, womit ich dich beehre, werth. - Sophia, du hast nun in kurzer Frist ein Leben voll Erfahrung gewonnen" (ebd.: 121). Ein Donner endet die Metamorphose, und die nun weise Sophia wird schlagartig zur Königin.

Der Erzähler des Märchens, Albert, stellt hier einen stark von männlichen Vorurteilen gegenüber gebildeten Frauen geprägten Erziehungsprozess dar. Letztlich ist das Dulden und Schweigen die für die erfolgreiche Eingliederung in die Gesellschaft entscheidende Prüfung, während weibliche Gelehrsamkeit und die Beschäftigung mit Philosophie gezielt als eitles Nachäffen der Gedanken männlicher Lehrer diskreditiert und die Bildung des Verstandes der Bildung des Herzens untergeordnet wird. Dementsprechend reserviert reagieren seine Zuhörerinnen im abendlichen Literaturzirkel, was im folgenden Kapitel des Romans geschildert wird. Doch Albert präsentiert sich selbst, möglicherweise unbeabsichtigt, ebenfalls als ein Erzähler, der nur der simplen Anspielung fähig ist, denn seine vielen philosophischen, pädagogischen und kulturgeschichtlichen Referenzen haben oftmals auch nur den Zweck des bloßen Scheins oberflächlicher Gelehrsamkeit. Das von ihm in Märchenform Kritisierte muss er sich letztlich selbst vorwerfen lassen.

4 Kammler (1992: 95) verweist auf den Kant-Kritiker Johann Georg Schlosser als möglichen Bezugspunkt für den Erfahrungsbegriff in Ungers Märchen. 


\section{Literatur}

d'Aulnoy, Marie-Catherine (2004): Babiole. In: Nadine Jasmin (Hg.): Contes des Fées suivis des Contes nouveaux ou Les Fées à la Mode. Paris: Champion, 507-533.

Baader, Renate (1986): Dames de lettres. Autorinnen des preziösen, hocharistokratischen und "modernen“ Salons (1649-1698): Mlle de Scudéry - Mlle de Montpensier - Mme d'Aulnoy. Stuttgart: Metzler.

Birberick, Anne L. (2011): Gendering Metamorphosis in d'Aulnoy’s “Babiole”. In: Seventeenth Century French Studies (33/2), 93-102.

Blamires, David (2006): Die deutsche Rezeption der Märchen von Marie-Catherine d'Aulnoy. In: Konrad Feilchenfeldt (Hg.): Zwischen Aufklärung und Romantik. Neue Perspektiven der Forschung. Festschrift für Roger Paulin. Würzburg: Königshausen \& Neumann, 138-149.

Borgards, Roland (2009): Affenmenschen/Menschenaffen. Kreuzungsversuche bei Rousseau und Bretonne. In: Michael Gamper (Hg.): „Es ist nun einmal zum Versuch gekommen. “Experiment und Literatur 1580-1790. Göttingen: Wallstein, 293-308.

Buffon, Georges-Louis Leclerc de (1791): Herrn von Buffons Naturgeschichte der vierfüßigen Thiere. Aus dem Französischen übersetzt, mit Anmerkungen, Zusätzen und Kupfern vermehrt, durch Bernhard Christian Otto. Bd. 17. Berlin: Pauli.

Dammann, Günter (1981): Conte de(s) fées. In: Rolf Wilhelm Brednich (Hg.): Enzyklopädie des Märchens. Handwörterbuch zur historischen und vergleichenden Erzählforschung. Bd. 3. Berlin: De Gruyter, Sp. 131-149.

Giesler, Birte (2003): Literat(ur)sprünge. Das erzäblerische Werk von Friederike Helene Unger. Göttingen: Wallstein.

Giesler, Birte (2009): Social Satire, Literary Parody, and Gender Critique in French and German Fairy Tales of the Enlightenment: Jean-Jacques Rousseau and Friederike Helene Unger. In: Andreas Böhn, Gaby Pailer (Hg.): Gender and Laughter. Comic Affrmation and Subversion in Traditional and Modern Media. Amsterdam: Rodopi, 233-250.

Giesler, Birte (2015): „... nie gerieth ein Mann, den nicht ein geliebtes Weib bildete.“ Weibliche literarische Kreativität als Kritik am Bildungsroman in Friederike Helene Ungers Prinz Bimbam. In: Linda Dietrick, Birte Giesler (Hg.): Weibliche Kreativität um 1800. Women's Creativity around 1800. Hannover: Wehrhahn, 99-123.

Griem, Julika (2010): Monkey Business. Affen als Figur anthropologischer und ästhetischer Reflexion 1800-2000. Berlin: trafo.

Hoffman, Kathryn (2005): Of Monkey Girls and a Hog-Faced Gentlewoman: Marvel in Fairy Tales, Fairgrounds, and Cabinets of Curiosities. In: Marvels \& Tales: Journal of Fairy-Tale Studies (19/1), $67-85$.

Janson, Horst (1952): Apes and Ape Lore in the Middle Ages and the Renaissance. London: The Warburg Institute at the University of London.

Kammler, Eva (1992): Zwischen Professionalisierung und Dilettantismus. Romane und ibre Autorinnen um 1800. Opladen: Westdeutscher Verlag.

Lehmstedt, Mark (1996): „Ich bin nun vollends zur Kaufmannsfrau verdorben.“ Zur Rolle der Frau in der Geschichte des Buchwesens am Beispiel von Friederike Helene Unger (1751-1813). In: Leipziger Jabrbuch zur Buchgeschichte (6), 81-154. 
Plinius Secundus, Gaius (1999): Naturkunde. Lateinisch-Deutsch. Bücher XXI/XXII. Hg. und übersetzt von Roderich König und Gerhard Winkler. Zürich/Düsseldorf: Artemis \& Winkler.

Rousseau, Jean-Jacques (1997): Diskurs über die Ungleichheit. Discours sur l'inégalité. Kritische Ausgabe des integralen Textes. Mit sämtlichen Fragmenten und ergänzenden Materialien nach den Originalausgaben und den Handschriften neu ediert, übersetzt und kommentiert von Heinrich Meier. Paderborn: Schöningh.

Seifert, Lewis C. (2011): Animal-Human Hybridity in d'Aulnoy's Babiole and Prince Wild Boar. In: Marvels \& Tales: Journal of Fairy-Tale Studies (25/2), 244-260.

Unger, Friederike Helene (1804): Albert und Albertine. Berlin: Unger.

Zantop, Susanne (Hg.) (1996): Friederike Helene Unger: Albert und Albertine. Mit einem Nachwort von Susanne Zantop. Hildesheim: Olms. 